\title{
Negative Mass: Part One
}

\section{Edward Tannous}

Jaffa, Israel

Email: Etannouss@gmail.com

How to cite this paper: Tannous, E. (2019) Negative Mass: Part One. Journal of Modern Physics, 10, 861-880.

https://doi.org/10.4236/jmp.2019.107057

Received: May 16, 2019

Accepted: June 27, 2019

Published: June 30, 2019

Copyright @ 2019 by author(s) and Scientific Research Publishing Inc. This work is licensed under the Creative Commons Attribution International License (CC BY 4.0).

http://creativecommons.org/licenses/by/4.0/

\begin{abstract}
This article presents a physical model, which describes the ideas of special relativity, in a rational, logical, simple and understandable manner, while using basic mathematical tools. The model is based on Albert Einstein's formula, which describes the "rest" energy of a body with mass $(m)$, given by the formula $E=m c^{2}$. Based on this formula, and in accordance with the theory of special relativity, we present here a model of a body, moving at a constant velocity in space (at high speeds, close to the speed of light), with speed equal to the speed of light in space-time, determined with an "energy angle" and negative mass. This model offers a method for creating negative mass, a calculating method for the relative velocity, and a method for calculating energy and momentum, in a completely elastic collision and plastic collision, different than in the contemporary nowadays method found in classical and modern physics. In addition, the new model solves problems and paradoxes known in special relativity physics, such as the Twin Paradox and others.
\end{abstract}

\section{Keywords}

Negative Mass, Energy Angle, Frame of Reference, Speed of Light, Special Relativity

\section{Introduction}

As known today in modern physics, and especially in the theory of special and general relativity, in quantum physics and cosmology, the Albert Einstein's formula describes the "rest" energy of a body with mass $(m)$, by the formula $E=m c^{2}$, which means that a body with mass $m$ has a proportional amount of energy and vice versa [1]. This formula determines that this energy $E$ can be calculated as the mass $m$ multiplied by the squared speed of light $\left(c=3 \times 10^{8} \mathrm{~m} / \mathrm{s}\right)$. The equation claims that if the body is stationary, it has an inner energy, which is its "rest" energy, in accordance with its rest mass. When the body is in motion, its total energy is greater than its rest energy, that is, its 
total mass is greater than its rest mass. Another assumption is regarding the speed of light. When the body moves, it can obtain momentum and energy, but when it approaches the speed of light, it cannot overpass it, regardless of the additional energy it receives [1].

Moreover, regarding the transition from one inertial frame of reference to another, Galileo's relativity assumption requires the principle of equivalence that the laws of motion are the same in all inertial reference frames [2].

Lorentz transformations, appearing in the theory of special relativity, are linear transformations of coordinates between two reference frames moving at a relative constant velocity and showing how time and space change when moving from one reference frame to an inertial reference frame which moves relatively to it at a constant velocity in a straight line [3]. Minkowski's diagrams [4] describe the space-time dynamics from a perspective of relativity, and make it possible to draw graphically and quite easily how a particular event (or collection of events) will be labeled in different frames.

It is well known that there are a few problems in the theory of special relativity, problems without a solution or with a solution which is not intuitively understood. For example, the Twin Paradox is a thought experiment in which a difference of age between a pair of twins is created: the first twin departs from his brother in space at a very high velocity and returns to Earth, while the second remains on Earth. When they meet again, the twin who left and returned back will be younger than his brother. The reason for the different period of time experienced by the twins is that they have undergone different paths in space-time between these two events. The "self-time" between two events depends on the path that connects them. Therefore, each of them measures another self-time. The solution to the Twin Paradox is difficult to understand and is explained by special relativity.

Another conceptual problem in physics, in general and in special relativity in particular, is that mass, energy, and time must have positive values and cannot have a negative value. As a result, there is currently no way to create a negative mass, there is no way to go back in time, and there is no way to produce gravitational repulsion forces.

Furthermore, the current cosmological measurements support the observation that the universe is expanding and its expansion rate is even increasing. The expansion of the universe is a term that describes the growth of distances between different points in the universe in time. One of the explanations for the expansion of the accelerating universe is based on the speculation of a dark unobserved energy, whose origins and properties are still not known.

The purpose of this paper is to provide a model of a body that explains in a deeper way the problems mentioned above. The model gives the same results of the special relativity in classical and modern physics, but in a simpler and easier way to understand, including Lorentz transformations [5], the Minkowsky time interval [6], the Doppler relativity effect, the Twin paradox, energy formulas, elastic and plastic collisions. Moreover, this article shows the existence of the 
concepts of the "energy angle" and negative mass, which can change the understanding of laws of physics as known today. The model makes the theory of special relativity understood in a more comprehensive way than before.

The new model and its definitions are described in Section 2. The building of the model and the application of the model formulas in the various fields are described in Section 3, including the solution of the problems mentioned above. Discussion, summary and conclusions are given in Section 4.

\section{A body Model Moving in the Space-Time}

The basic premise of this new theory is that each body or particle moves in space-time at the speed of light, which is derived from the formula $E=m c^{2}$. A body with mass $m_{1}$ in space observes the universe in a different way, with different dimensions and times as compared to another body with a mass $m_{2}$, moving at a different velocity. Moreover, each body feels at rest even if its speed is close to the speed of light relative to another body.

The proposed theory is also based on the Galilean equivalence principle, which claims that the laws of physics are the same in all inertial reference frames. However, instead of the assumption of the constancy of the speed of light, the theory here states that the energy of each mass $m$ in any reference frame is constant, i.e. $E=m c^{2}$, and each mass $m$ measured in any spatial-temporal reference frame of four dimensions is already moving at the speed of light $c$.

When the body is at rest in the Euclidean space $(x, y, z)$, it can be said that it moves in the fourth dimension which is the time axis $(t)$, and its speed is the speed of light. This time axis is called "Light Time Axis" and we mark it as (ct). The "light time axis" is orthogonal to the three axes in the Euclidean space $(x, y, z)$.

The speed of light is the only absolute speed, to all bodies in the universe, and any other speed is actually the projection of the speed of light on the spatial axes, or the difference between the speeds of light in different directions in the frame axes of space-time.

Each body will be have "self-speed of light vector" $\boldsymbol{C}$, where each body with mass has its own self-speed of light vector in its four-dimensional space $\boldsymbol{C}=(c, 0,0,0)$ and each body has a different direction of the 'self-speed of light vector' if we observe it from a common frame of reference, unless they are at the same speed and in the same direction, i.e., the two bodies are at rest relative to each other. In this case, their "self-speed of light vector" will be equal, regardless of the magnitude of the mass.

The 'self-speed of the light vector' has the following properties:

1) The modulus of the "self-speed of light vector" is always equal to the speed of light: $|\boldsymbol{C}|=\left|\boldsymbol{C}^{\prime}\right|=C$

2) At any given time $t$, "the light distance" measured in the frame of reference is equal for all the masses in any frame: $\left|\boldsymbol{C}_{1} \Delta t\right|=\left|\boldsymbol{C}_{2} \Delta t\right|=\cdots$

3) The projection of the "self-speed light vector" on the Euclidean space velocity axis $(x, y, z)$ gives a velocity $\boldsymbol{v}^{\prime}$ and the projection on the time axis 
gives its self-time velocity $v_{t}^{\prime}$, wherein $c^{2}=v_{t}^{\prime 2}+v_{x}^{\prime 2}+v_{y}^{\prime 2}+v_{z}^{\prime 2}$.

4) When the body moves in a Euclidean frame of reference $(x, y, z)$ at a velocity $\boldsymbol{v}^{\prime}=\left(v_{x}^{\prime}, v_{y}^{\prime}, v_{z}^{\prime}\right)$, the "self-speed light vector" is $\boldsymbol{C}^{\prime}=\left(v_{t}^{\prime}, v_{x}^{\prime}, v_{y}^{\prime}, v_{z}^{\prime}\right)$. The "self-time Velocity" $v_{t}^{\prime}=\frac{\mathrm{d}\left(c t^{\prime}\right)}{\mathrm{d} t}$ of the moving body in a frame of reference can be calculated, as: $v_{t}^{\prime 2}=c^{2}-\left(v_{x}^{\prime 2}+v_{y}^{\prime 2}+v_{z}^{\prime 2}\right)$, or by the projection of the vector $\boldsymbol{C}^{\prime}$ on the self-speed light vector $\boldsymbol{C}$ of the frame of reference $v_{t}^{\prime}=\frac{\boldsymbol{C} \cdot \boldsymbol{C}^{\prime}}{C}$

5) The "energy angle" $\alpha$ is the angle between two self-speed light vectors of the reference frame with respect to the moving one, therefore: $\sin \alpha=\frac{v}{c}$ where $v=\left|v^{\prime}\right|=\sqrt{v_{x}^{\prime 2}+v_{y}^{\prime 2}+v_{z}^{\prime 2}}$ or $v_{t}^{\prime}=c \cdot \cos \alpha$.

The properties of the self-speed of the light vector are obtained as a result of simple basic mathematics, i.e. it is based on its definition as a vector representing velocity.

\section{Evolution of a Body Moving in the Space-Time}

In this section the evolution of a body moving at the speed of light in the space-time, in accordance with this new theory and the model derived from it will be shown. The formulas derived from this new theory, and its implications and applications in the various branches of physics, including solutions of special problems in physics will be detailed.

\subsection{Development of Equations According to the Model}

This sub-section presents the equations which are derived from this new theory. At first we refer to one body and its relation to other bodies will be discussed later. The evolution of the body in terms of speed, energy, energetic angle, and from there how to obtain a negative mass will be treated. From there it will be shown how to obtain known formulas in physics via this new theory, which include energy formulas, specifically for completely elastic collision and plastic collisions.

\subsubsection{One-Dimensional Space}

Without losing generality, reference will be made to only a one-dimensional spatial frame (1D) in $x$ and $x^{\prime}$ axes, orthogonal to $c t$ and $c t^{\prime}$, respectively. The 1D choice is only to ease the understanding of the theory. The theory applies to 3D models as well.

Figure 1(a) describes the velocity frame of reference of a body moving in relation to a frame of reference at speed $v$ in direction $x$, showing a self-speed light vector of the moving body, consisting of two elements, the velocity $v$ in the direction of $x \quad v=v_{x}=\mathrm{d} x / \mathrm{d} t$ and the velocity along the time axis $v_{t}^{\prime}$ of the mass in the direction of the axis of the self-speed of light vector of the reference frame. $C$ is the "self-speed light vector" in the frame of reference and $C^{\prime}$ is the "self-speed light vector" of another moving body. The angle $\alpha$ describes the rotation angle between the two frames. The projection of the self-speed light of the 
moving body $C^{\prime}$ on the horizontal axis $v_{x}$ gives the velocity $v$ in the $x$ direction. Its projection on axis $\boldsymbol{C}$ gives the self-time speed $v_{t}^{\prime}=\mathrm{d}\left(c t^{\prime}\right) / \mathrm{d} t$, which is the speed of light time of the moving body measured in the frame of reference.

The $x$ or $x^{\prime}$ axis is determined perpendicularly to the "self-speed of light vector" of each frame separately. Thus, we obtain that the angle $\alpha$ between the two "self-speed light vectors" is the same angle between the two axes $x$ and $x^{\prime}$.

Figure 1(b) describes a model called "Integral Couple Frame" (ICF), a time integration frame with the following meaning:

An integral on $v_{x}$ creates the $x$-axis and its direction.

An integral on $C^{\prime}$ creates the $c t$ axis and its direction.

An integral on $v_{t}^{\prime}$ creates the $c t^{\prime}$ axis and its direction.

It should be noted that the $x^{\prime}$ axis is normalized to the "self-speed light vector" of the moving body $C^{\prime}$.

The result gives two frames with distances $x^{\prime}, c t$ and $x, c t^{\prime}$ wherein the ct time axis is not normal to the $x$ axis, and the $c t^{\prime}$ axis is not normal to the $x^{\prime}$ axis.

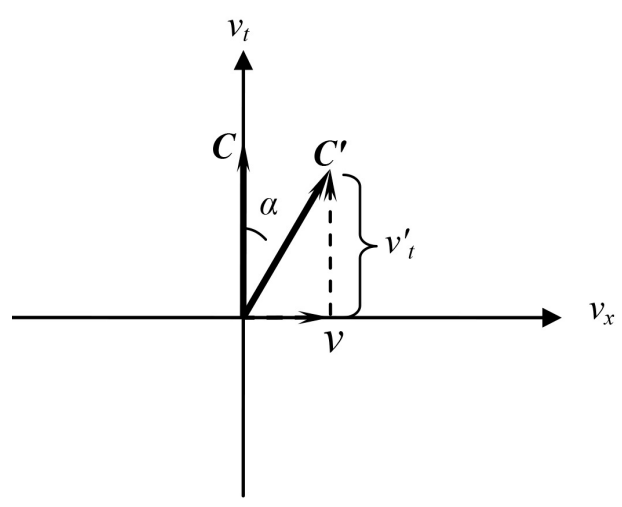

(a)

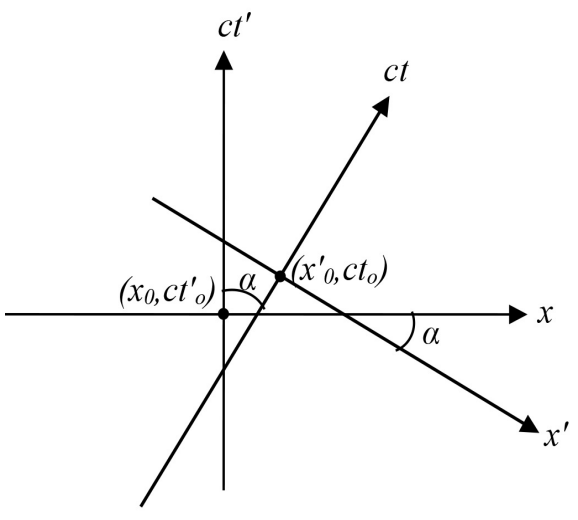

(b)

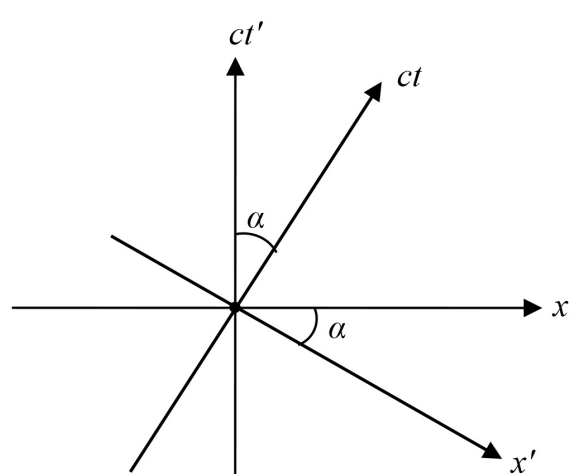

(c)

Figure 1. (a) The velocities plot of the moving body model relative to a frame of reference, with $\boldsymbol{C}$ "self-speed of light vector" in the body frame of reference and $\boldsymbol{C}^{\prime}$ the "self-speed of light vector" of the moving body. The angle $\alpha$ describes the rotation angle between the two frames;. (b) "Integral Couple Frame" Model (ICF), which is a time integra- 
tion. The angle $\alpha$ is the same angle as in Figure 1(a); (c) The body's initial condition.

The parameters $x_{0}, x_{0}^{\prime}, c t_{0}, c t_{0}^{\prime}$ are the constants obtained from the integration operation and have values that can be calculated according to the boundary values, i.e. the initial conditions. The main axes can be placed one over the other and can be reset in time and space as shown in Figure 1(c). The ICF model, which describes the intersection between $x^{\prime}$ and $c t$ axes, shows the motion in the frame with time progression, while the point of $x^{\prime}=0$ will always be attached to the $c t$ value.

\subsubsection{Lorentz Transformation}

As mentioned above, the value of the angle $\alpha$ depends proportionally on the magnitude of the speed $v$ :

$$
v=c \sin \alpha
$$

The parameter $\beta$ can be found from Equation (1), that is the ratio between the speed $v$ and the speed of light $c$ :

$$
\beta=\frac{v}{c}=\sin \alpha
$$

The Lorentz factor defined in Einstein's theory of relativity and its relation to the energetic angle $\alpha$ can also be identified here as:

$$
\gamma=\frac{1}{\sqrt{1-\frac{v^{2}}{c^{2}}}}=\frac{1}{\sqrt{1-\sin ^{2} \alpha}}=\frac{1}{\cos \alpha}
$$

Figure 2 describes the changes in distances and light time in the moving frame and their projections in the stationary frame. The differences in the moving frame measured in the reference frame are:

$$
\begin{aligned}
\Delta x & =\cos \alpha \cdot \Delta x^{\prime} \\
\Delta t & =\frac{1}{\cos \alpha} \cdot \Delta t^{\prime}
\end{aligned} \text { therefore } \begin{aligned}
\Delta x & =\frac{1}{\gamma} \Delta x^{\prime} \\
\Delta t & =\gamma \Delta t^{\prime}
\end{aligned}
$$

In the equations above, two concepts are obtained, Length contraction and Time dilation. That is, the length $\Delta x^{\prime}$ of the moving frame measured from the reference frame $\Delta x$ is shorter. In fact, the length and time of light do not change, but their projections from one frame to another are different.

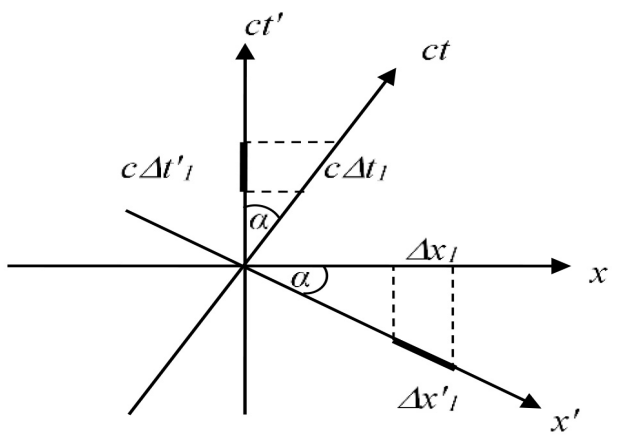

Figure 2. The changes in distance and light time in the moving frame and their projections in the stationary frame. 
Figure 3 describes two events, $e$ and $k$, in the $x, c t$ plane and $x^{\prime}, c t^{\prime}$ plane and the projections of the coordinates in both frames. The values of the events $e$ and $k$ are obtained by calculating the coordinate's projections of the events in one frame onto the other frame, and the subtraction between them results in the equations of the following differences.

i.e. $\Delta x=x_{e}-x_{k}, \Delta x^{\prime}=x_{e}^{\prime}-x_{k}^{\prime}, \Delta c t=c t_{e}-c t_{k}$ and $\Delta c t^{\prime}=c t_{e}^{\prime}-c t_{k}^{\prime}$.

We obtain thus:

$$
\begin{array}{ll}
\Delta x^{\prime}=\cos \alpha \cdot \Delta x-\sin \alpha \cdot c \Delta t^{\prime} \\
c \Delta t=\cos \alpha \cdot c \Delta t^{\prime}+\sin \alpha \cdot \Delta x
\end{array} \text { and vice versa } \begin{aligned}
& \Delta x=\cos \alpha \cdot \Delta x^{\prime}+\sin \alpha \cdot c \Delta t \\
& c \Delta t^{\prime}=\cos \alpha \cdot c \Delta t-\sin \alpha \cdot \Delta x^{\prime}
\end{aligned}
$$

We can substitute Equation (1) and (3) into Equation (5), resulting in Lorentz transformation:

$$
\begin{array}{r}
\Delta x^{\prime}=\gamma(\Delta x-v \Delta t) \text { and vice versa } \Delta x=\gamma\left(\Delta x^{\prime}+v \Delta t^{\prime}\right) \\
\Delta t^{\prime}=\gamma\left(\Delta t-\frac{v}{c^{2}} \Delta x\right) \quad \Delta t=\gamma\left(\Delta t^{\prime}+\frac{v}{c^{2}} \Delta x^{\prime}\right)
\end{array}
$$

If the distance between the two events $e$ and $k$ is $l$, Minkowski's interval formula can be obtained directly without using an imaginary time axis:

$$
\begin{gathered}
l^{2}=(c \Delta t)^{2}+\Delta x^{\prime 2}=\left(c \Delta t^{\prime}\right)^{2}+\Delta x^{2} \\
\Downarrow \\
\Delta s^{2}=(c \Delta t)^{2}-\Delta x^{2}=\left(c \Delta t^{\prime}\right)^{2}-\Delta x^{\prime 2}
\end{gathered}
$$

In the Euclidean space $x, y, z$ :

$$
\Delta s^{2}=(c \Delta t)^{2}-\Delta x^{2}-\Delta y^{2}-\Delta z^{2}=\left(c \Delta t^{\prime}\right)^{2}-\Delta x^{\prime 2}-\Delta y^{\prime 2}-\Delta z^{\prime 2} .
$$

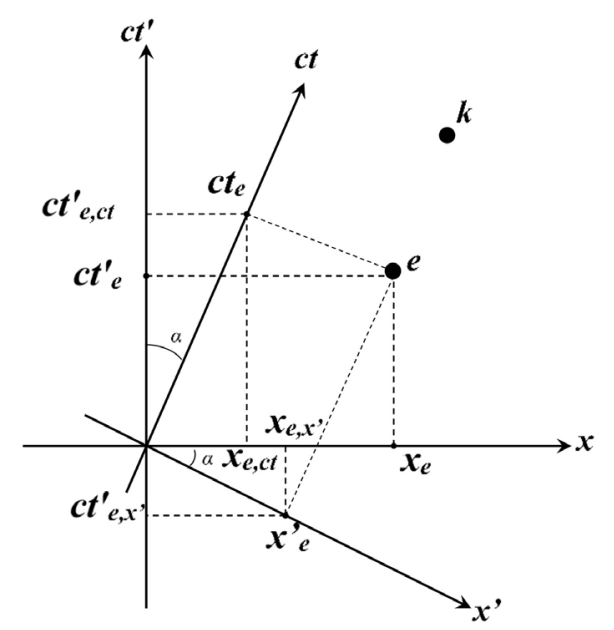

Figure 3. Two events $e$ and $k$ in the $x, c t$ plane and $x^{\prime}, c t^{\prime}$ plane and coordinates' projections for both frames.

\subsubsection{Invariance of the Speed of Light in the 2 Frames}

This theory, as we have pointed out, is based on one premise, namely that each body moves in space-time at the speed of light. Here we will demonstrate that 
Einstein's premise, which is that the speed of light is equal in all frames, is indeed obtained from our premise regarding the energy of a moving body.

Figure 4(a) describes a photon exiting from the origin of the stationary frame at time $t=t^{\prime}=0$ and making its way to point $x_{1}$. The angle at which the photon advances in the $x, c t$ plane is $\beta$, which is a bisector of the two axes i.e., $\beta=(\pi / 4-\alpha / 2)$.

Figure 4(b) describes the photon reaching point $x_{1}$, where the stationary frame progresses in time to a value of $c t_{1}$. The values of $x^{\prime}$ and $c t^{\prime}$ are equal at this point. Both the stationary and moving frames measure a speed of light equal to $c$, even though the distances and times are not equal. The ratio of the distances that the light travels in the stationary and moving frames is:

$\frac{x^{\prime}}{x}=\frac{c t^{\prime}}{c t}=\tan \beta=\frac{1-\sin \alpha}{\cos \alpha}$ which shows a Doppler effect for the relativistic case.

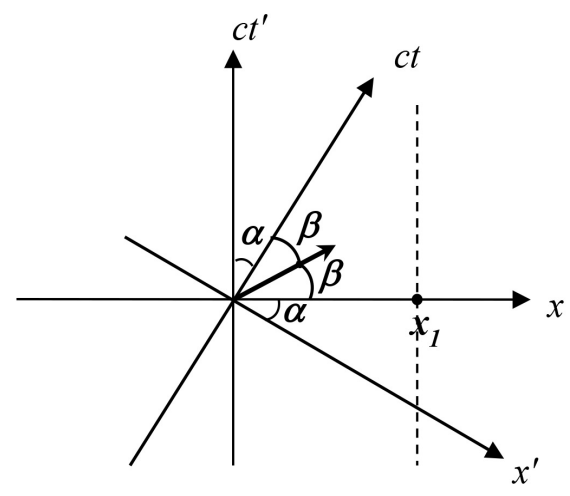

(a)

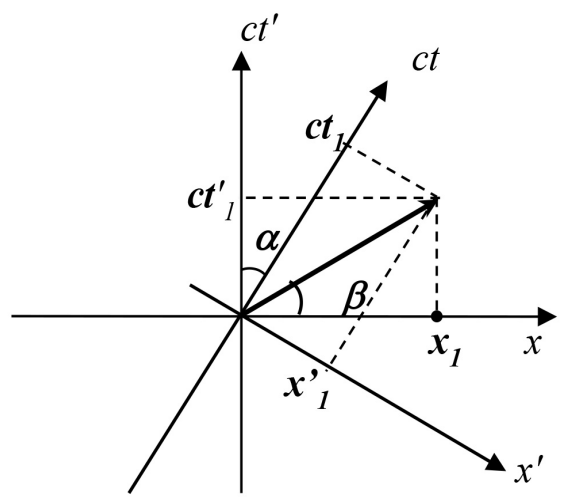

(b)

Figure 4. (a): Scheme of a photon exiting from the origin of the stationary frame and the description of distances and times in the frames. (b): The photon reaches point $x_{l}$ and the description of the distances and times in the frames.

Figure 5 describes the separation velocity vector $\boldsymbol{V}_{a x}$, the separation of the axes' origin of the moving frame $\left(x^{\prime}, y^{\prime}, z^{\prime}\right)=(0,0,0)$ from the axes' origin of the stationary (reference) frame $(x, y, z)=(0,0,0)$ in the space-time plane. The velocity of the moving frame is the vector difference between two vectors of the speed light vectors of both frames with $\boldsymbol{C}_{1}$ and $\boldsymbol{C}_{2}$. The vectors in the polar description $(r, \theta)$ are: $C_{1}=\left(c, \frac{\pi}{2}\right)$ and $C_{2}=\left(c, \frac{\pi}{2}-\alpha\right)$, whose representation in Cartesian form is: $C_{1}=(0, c)=c \hat{v}_{t}$ and $C_{2}=\left(v, v_{t}^{\prime}\right)=v \hat{v}_{x}+v_{t}^{\prime} \hat{v}_{t}$ wherein: $\hat{v}_{t}$ is a unit vector of the velocity in the direction of the "light time" axis $c t$, and $\hat{v}_{x}$ is a unit vector velocity in the direction of the $x$-axis. $\boldsymbol{V}_{a x}$ is the separation velocity vector, i.e., the separation of the two bodies in the space-time, whose direction is the movement of the axes' origin of the one-dimensional moving frame $\left(x^{\prime}\right)=(0)$ relative to the axes' origin of the stationary frame 
$(x)=(0)$.

Using $v=c \sin \alpha$ and $v_{t}^{\prime}=c \cdot \cos \alpha$ will lead to $\boldsymbol{V}_{a x}=c \sin \alpha \hat{v}_{x}+c(\cos \alpha-1) \hat{v}_{t}$

The use of $\cos \alpha=\cos ^{2}(\alpha / 2)-\sin ^{2}(\alpha / 2)$ and $\sin \alpha=2 \sin (\alpha / 2) \cos (\alpha / 2)$ will lead to: $\boldsymbol{V}_{a x}=2 c \sin (\alpha / 2) \cos (\alpha / 2) \hat{v}_{x}+c\left(\cos ^{2}(\alpha / 2)-1-\sin ^{2}(\alpha / 2)\right) \hat{v}_{t}$

Finally using $\cos ^{2}(\alpha / 2)-1=-\sin ^{2}(\alpha / 2)$ will lead to:

$$
\begin{gathered}
\boldsymbol{V}_{a x}=2 c \sin (\alpha / 2) \cos (\alpha / 2) \hat{v}_{x}-2 c \sin ^{2}(\alpha / 2) \hat{v}_{t} \\
\boldsymbol{V}_{a x}=2 c \sin (\alpha / 2)\left(\cos (\alpha / 2) \hat{v}_{x}-\sin (\alpha / 2) \hat{v}_{t}\right)
\end{gathered}
$$

In a polar representation the upper expression, gives the following formula:

$$
\boldsymbol{V}_{a x}=\boldsymbol{C}_{2}-\boldsymbol{C}_{1}=\left(2 c \sin \left(\frac{\alpha}{2}\right),-\frac{\alpha}{2}\right)
$$

The projection of the velocity vector on the $x$-axis gives the relation for the speed $v$ using Equation (1):

$$
V_{a x} \cos \frac{\alpha}{2}=2 c \sin \frac{\alpha}{2} \cos \frac{\alpha}{2}=c \sin \alpha=v
$$

When the moving frame reaches the speed $v=c$, the value of $\boldsymbol{V}_{a x}$ will be greater than the speed of light, in the space-time, and its angle is $-45^{\circ}$. Thus, when $\alpha=\pi / 2, \quad V_{a x}=\left(\sqrt{2} \cdot c,-\frac{\pi}{4}[\mathrm{rad}]\right)$. This result does not contradict the known laws of physics nowadays, namely that the transfer of energy or mass cannot exceed the speed of light in the Euclidean frame $(x, y, z)$ or $\left(x^{\prime}, y^{\prime}, z^{\prime}\right)$. Indeed, this velocity is in the space-time, and by its projections on each of the three axes, a speed is obtained, which is not greater than the speed of light.

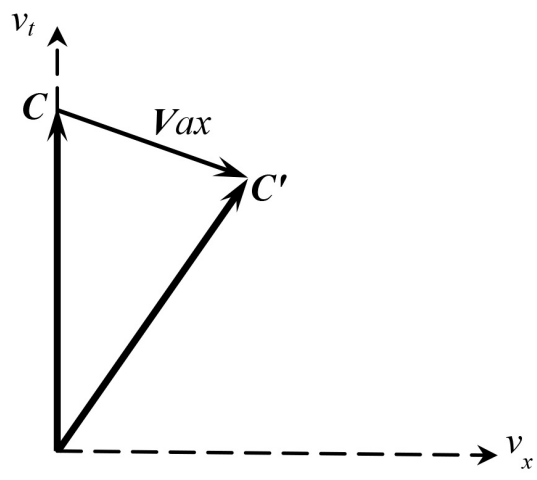

Figure 5. The separation velocity vector, is the separation of the moving frame from the stationary frame.

Figure 6 describes the model for calculating relative velocities of three bodies $1,2,3$ at different velocities. The first body is treated as a reference frame 1 with $x_{1}, c t_{1}$, i.e. $v_{11}=0$, wherein the speed of body number 1 relative to frame 1 is zero, and the self-speed of light vector is $C_{1}=\left(c, \frac{\pi}{2}\right)$. The second body's frame 
$x_{2}, c t_{2}$ moves at a speed $v_{12}$ relative to the first frame and the angle $\alpha_{12}$ is: $\sin \alpha_{12}=v_{12} / c$ with a self-speed of light vector $C_{2}=\left(c, \frac{\pi}{2}-\alpha_{12}\right)$, whose projection on the $x$ axis is $v_{12}$. The velocity of the third body relative to the second frame is $v_{23}$ (we assume that the velocity is in the positive direction of $x$ ) with the angle $\alpha_{23}$ according to $\sin \alpha=v_{12} / c$ and gives the self-speed of light vector $\boldsymbol{C}_{3}=\left(c, \frac{\pi}{2}-\alpha_{23}\right)$. The velocity of the third body $v_{13}$ by projection of its self-speed of light $\boldsymbol{C}_{3}$ on the $v_{x 1}$ axis of the reference frame is:

$$
v_{13}=c \sin \left(\alpha_{12}+\alpha_{23}\right)
$$

At speeds much smaller than the speed of light, we obtain $v_{13}=v_{12}+v_{23}$, following Equation (1) since: $\lim _{\alpha_{12}, \alpha_{23} \rightarrow 0} \sin \left(\alpha_{12}+\alpha_{23}\right)=\sin \alpha_{12}+\sin \alpha_{23}$.

The velocity of the third frame in the reference frame can also be calculated if the velocity $v_{23}$ is in the direction of $y_{2}$ axis of the second frame. We therefore obtain the following expression: $\cos \alpha_{13}=\cos \alpha_{12} \cdot \cos \alpha_{23}$. It is possible to calculate $v_{13}$ from Equationn (1) by: $v_{13}^{2}=v_{12}^{2}+v_{23}^{2}-\frac{v_{12}^{2} v_{23}^{2}}{c^{2}}$. Here, too, at small velocities, we obtain $\lim _{v_{12}, v_{23} \ll c} v_{13}^{2}=v_{12}^{2}+v_{23}^{2}$ which is a vector addition of speeds according to Pythagoras theorem.

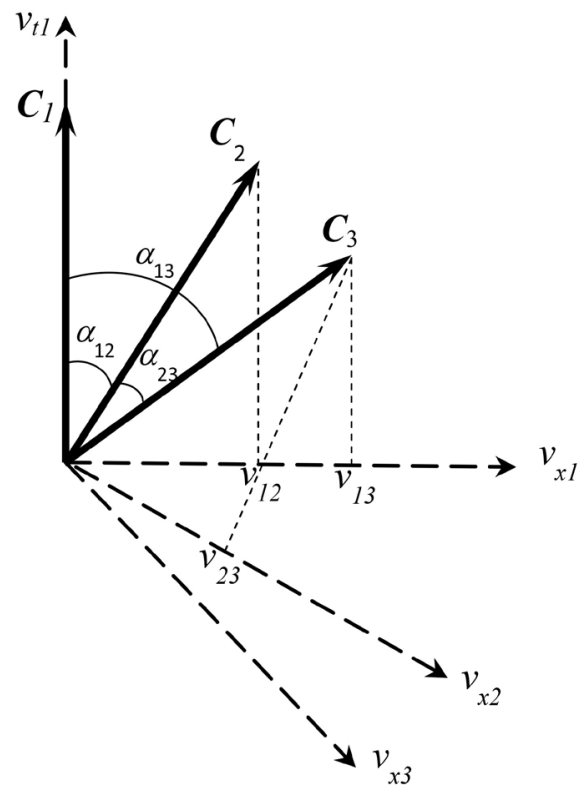

Figure 6. A model presentation for the calculation of relative velocities while describing frames of three bodies at different velocities, and the first body is a reference frame.

Figure 7 describes an array of self-speed of light vectors of three bodies, wherein body 2 moves in the direction of the $x$-axis and body 3 moves in the direction of the $y$-axis. 


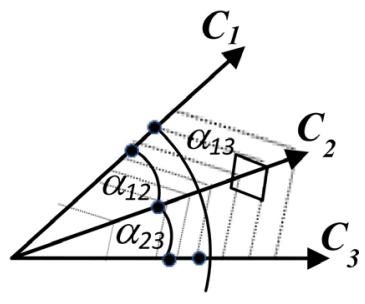

Figure 7. Vector description of self-speed of light vectors of three bodies.

\subsubsection{Energy}

In this model, the body moves always at the speed of light in space-time, and the velocity of the body in the Euclidean space $x, y, z$ does not change the value of the self-speed of light vector, but only its direction, i.e. the angle $\alpha$. Accordingly, its total energy is $m_{0} c^{2}$ ( $m_{0}$ is the rest mass of the body). The self-speed light vector in the fourth-dimensional space is: $C^{\prime}=\left(\frac{\mathrm{d}\left(c t^{\prime}\right)}{\mathrm{d} t}, v\right)$, which is composed of two main parts, the self-speed of light $v_{t}=\frac{\mathrm{d}\left(c t^{\prime}\right)}{\mathrm{d} t}$ and the speed of the body relative to the reference frame in the Euclidean space $v$.

The energy consists of the following components:

A) The body self-time energy $E_{s t}$, which determines the amount of mass left as a result of the movement.

B) "State" kinetic energy $E_{\alpha}$, which is the energy that the body carries in the reference frame, as a result of its speed. (In this case, this energy is kinetic energy, but in other cases, it may also include other energies, therefore here the sign and name are).

The self-time energy $E_{s t}$ is the product of the mass $m$ and $C_{1}$ and $C_{2}$ that is, the projection of the moving body's self-speed of light vector on the reference frame:

$$
E_{s t}=m_{0} \boldsymbol{C}_{1} \cdot \boldsymbol{C}_{2}=m_{0} c^{2} \cos \alpha \equiv \frac{m_{0} c^{2}}{\gamma}
$$

i.e., the self-time energy of the mass is smaller than the "rest" energy $E_{0}=m_{0} c^{2}$ reaching a value of $m_{0} c^{2} \cos \alpha$ of the energy in a moving condition, and the difference between them is actually the state kinetic energy $E_{\alpha}$, which can also be obtained by the projection of the velocity $C_{2}$ on the motion direction of the moving frame in the following formula:

$$
E_{\alpha}=m_{0} \boldsymbol{C}_{2} \cdot \boldsymbol{V}_{a x}=2 m_{0} c^{2} \sin ^{2}(\alpha / 2)=m_{0} c^{2}(1-\cos \alpha) \equiv m_{0} c^{2}\left(1-\frac{1}{\gamma}\right)
$$

For small values of $\alpha$ (small speeds relative to the speed of light), the state kinetic energy tends to $E_{k}=\frac{m_{0} v^{2}}{2}$ from $\lim _{\alpha \rightarrow 0} \frac{E_{\alpha}}{\frac{m_{0} v^{2}}{2}}=\frac{m_{0} c^{2}(1-\cos \alpha)}{\frac{m_{0} c^{2} \sin ^{2} \alpha}{2}}=1$ and when the speed approaches the speed of light, the energy approaches 
$E_{k}=E_{\alpha}=m_{0} c^{2}$.

That is, when $\alpha=\pi / 2$, the total energy of the mass is kinetic $m_{0} c^{2}$ and the self-time energy is zero at this point. This model is in contrast to Einstein's result, that the mass increases to infinity as the speed increases and approaches the speed of light, thus the formula he obtained $E=\gamma m_{0} c^{2}$ is incorrect according to our model.

According to the model of the current theory, the energy conservation law is kept and the sum of the two energies $E_{s t}$ and $E_{\alpha}$ is the rest energy of the body $E_{0}=m_{0} c^{2}$ :

$$
E_{0}=E_{s t}+E_{\alpha}=m_{0} c^{2}
$$

\subsubsection{Negative Mass}

Figure 8 describes quadrant zones of the changes in the energetic state of a moving body for changes of the energetic angle $\alpha$, in the range of $(-\pi)$ to $(\pi)$. Quadrant A shows the change in the velocity from 0 to $c$, quadrant $\mathrm{B}$ shows the change in the velocity from 0 to $-c$, quadrant $C$ shows the change in the velocity from $c$ to 0 and quadrant $\mathrm{D}$ shows the change in the velocity from $-c$ to 0 . Quadrants $C$ and $D$ are areas where the self-time speed is negative, so that the self-time obtained by the integration method (as shown in Figure 1) is negative, and it is a zone of negative mass.

Figure 9 describes the ratio of the speeds and energies of a body as a function of the energetic angle $\alpha$, in accordance with the measurement of the velocity and its direction in the reference frame. The sum of the body total energy is equal to $E_{0}=m_{0} c^{2}$. When the energy angle $\alpha$ is $\pi / 2$, the speed energy angle of the moving body reaches the speed of light and the state kinetic energy is equal to $m_{0} c^{2}$. With greater energy input, the body's speed will decrease until the energy angle reaches $\pi$ and the body will be in a rest state with state kinetic energy of $2 m_{0} c^{2}$ and self-time energy $\left(-m_{0} c^{2}\right)$. The figure shows the speed of a moving body normalized to $c$, the state kinetic energy $E_{\alpha}$ and the self-time energy $E_{s t}$ normalized both to $m_{0} c^{2}$, as a function of the angle $\alpha$ which extends in the range of $-\pi$ and $\pi$, from measurements in a stationary frame. Increasing the speed causes the body mass to be reduced. When the speed reaches the speed of light, the body will be completely massless. The self-time energy $E_{s t}$ is the amount of energy left in the body after some of it becomes kinetic energy, and its mass decreases by:

$$
m=m_{0} \cos \alpha=m_{0} / \gamma
$$

As the body reaches $\alpha=\pi / 2$, the speed begins to gradually decrease and the body reaches a negative self-time zone. When the body reaches $\alpha=\pi$, the speed is zero, the kinetic energy is $E_{k}=2 m_{0} c^{2}$ and the self-time energy is $E_{s t}=-m_{0} c^{2}$. The effective mass $m$ of the body (the mass which the reference frame perceives of the same body) is:

$$
E_{s t(\alpha=\pi)}=-m_{0} c^{2}=m_{(\alpha=\pi)} c^{2} \Rightarrow m_{(\alpha=\pi)}=-m_{0}
$$


The value of the gravitational force is in inverse ratio by:

$$
F_{(\alpha=\pi)}=G \frac{m_{(\alpha=\pi)} M}{r^{2}}=G \frac{-m_{0} M}{r^{2}}<0
$$

where in $M$ is the mass of the gravitational source (in the reference frame), $r$ is the distance between the masses centers and $G$ is the gravitational constant.

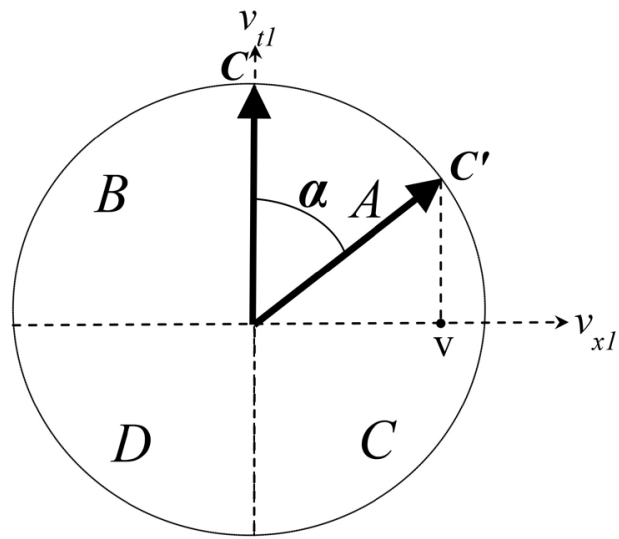

Figure 8. Zones of changes in the energetic state of a moving body with the change of the energetic angle $\alpha$, in the range of $(-\pi)$ to $(\pi)$. Quadrants $\mathrm{C}$ and $\mathrm{D}$ are zones where the self-time speed is negative, so that the self-time is negative, and it is a zone of negative mass.

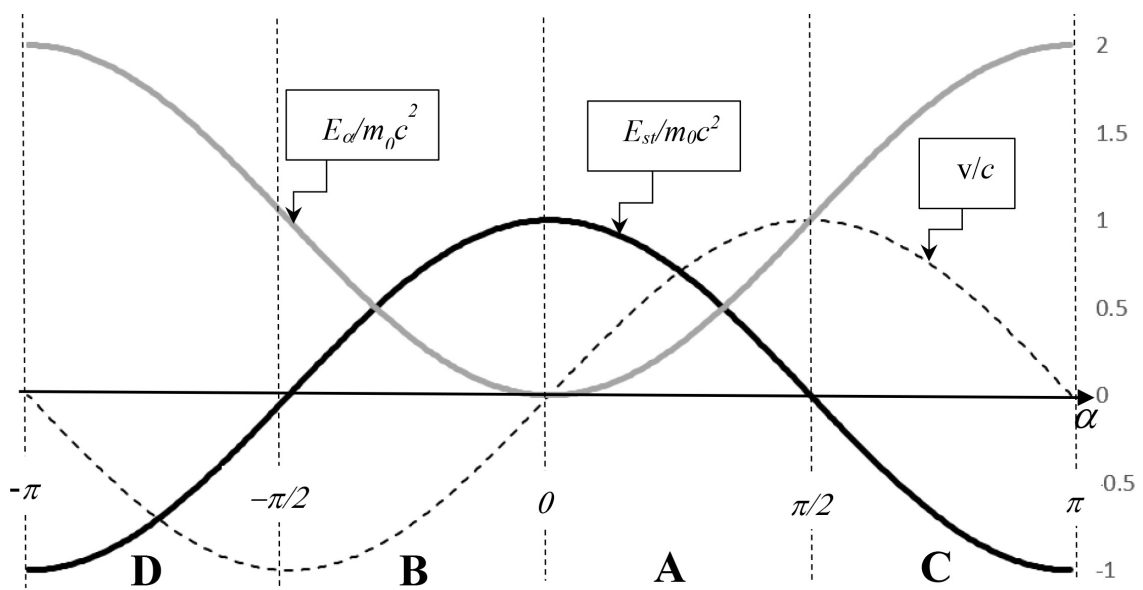

Figure 9. The ratio of the normalized speeds and energies of a body as a function of the energetic angle $\alpha$. The graph shows the speed of a moving body normalized to $c$, the kinetic energy $E_{k}$ normalized to $m_{0} c^{2}$, and the self-time energy $E_{s}$ normalized to $m_{0} c^{2}$, as a function of the angle $\alpha$ in the range of $-\pi$ and $\pi$ as measurements from a stationary frame.

Attention is now drawn to Figure 10(a) and Figure 10(b), when the body exceeds $\alpha=\pi / 2$. Figure 10(a) is in the range of $\pi / 2<\alpha<\pi$ and Figure 10(b) is in the range of $-\pi<\alpha<-\pi / 2$ When the body exceeds the speed-of-light barrier, its speed decreases and its self-time energy receives negative values. The 
self-time of the moving frame progresses in the negative direction. The projection of a body, moving in the direction of the arrow, in the $x$-axis is in reverse to its direction, i.e. it will move away. Its direction is reversed, therefore its future can be seen, but after a while it moves away and its past can be seen. When the body is in a gravitational field, it will move against gravity, moving from the future to the past.

Case $\pi / 2<\alpha<\pi$

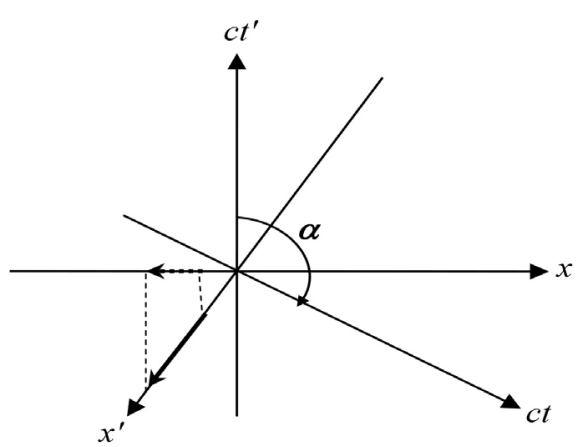

(a) case $-\pi<\alpha<-\pi / 2$

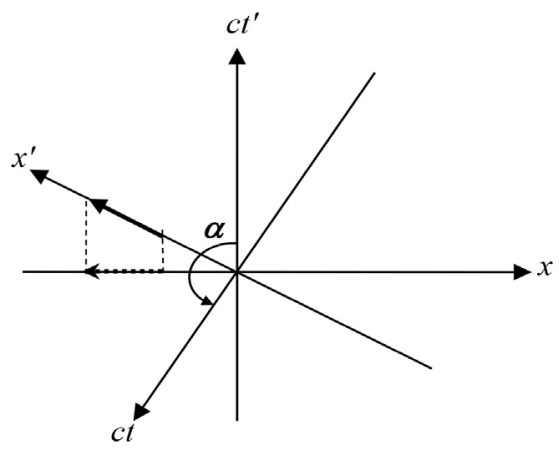

(b)

Figure 10. (a) Two frames model for the energy angles in the range of $\pi / 2<\alpha<\pi$; (b) Two frames model for the energy angles in the range of $-\pi<\alpha<-\pi / 2$.

Figure 11 shows several bodies $\mathrm{K}, \mathrm{L}, \mathrm{M}, \mathrm{N}$ with different self-velocity vectors and the forces of attraction or rejection between them, where body $\mathrm{K}$ represents the reference frame. There is an attraction force between the body $\mathrm{K}$ and body $\mathrm{L}$ and a repulsive force between body $\mathrm{K}$ and body $\mathrm{N}$. Also, there is an attraction force between body $\mathrm{L}$ and the two bodies, $\mathrm{K}$ and $\mathrm{N}$. When body $\mathrm{L}$ moves to the right at a speed close to the speed of light and body $M$ moves to the left at a speed close to the speed of light, the speed of body $\mathrm{L}$ relative to body $\mathrm{M}$ is significantly smaller than the speed of light (it can be seen from the projection of the self-speed of light vector on the $x$ axis of body M), because in relation to it, body $\mathrm{L}$ has a negative mass. A negative mass is relative to another mass when the angle between the self-speed vectors between the two masses is greater than $\pi / 2$. Negative mass is also obtained for values of velocities smaller than the speed of light, including the zero velocity (a state of rest).

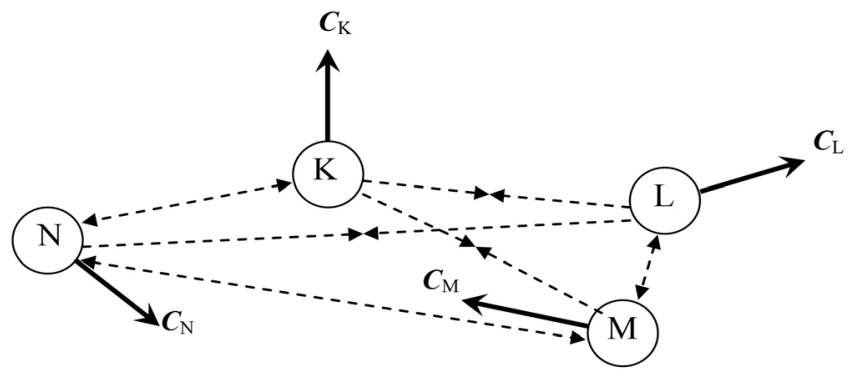

Figure 11. Description of bodies with different self-velocity vectors and the forces of attraction or repulsion between them. 


\subsubsection{The Amount of Energy Required to Bring a Body to the Speed of Light}

The model also provides a method for calculating the amount of energy required to bring a body from a state of rest to the speed of light, according to the following steps:

Applying an $F$ force in order to change the angle of the self-speed of light vector from $\alpha=0$ to $\alpha=\pi / 2$.

Inserting the momentum value $F=\frac{\mathrm{d} p^{\prime}}{\mathrm{d} t}$ in the formula $\mathrm{d} E_{\alpha}=F \mathrm{~d} x^{\prime}$ leads to $\mathrm{d} E_{\alpha}=\mathrm{d} p^{\prime} \underbrace{\frac{\mathrm{d} x^{\prime}}{\mathrm{d} t}}_{v^{\prime}}$

Using the momentum relation $p^{\prime}=m^{\prime} v^{\prime}$ and the differential $\mathrm{d} p^{\prime}=m^{\prime} \mathrm{d} v^{\prime}+v^{\prime} \mathrm{d} m^{\prime}$ will lead to $\mathrm{d} E_{\alpha}=m^{\prime} v^{\prime} \mathrm{d} v^{\prime}+v^{\prime 2} \mathrm{~d} m^{\prime}$.

Using Equation (1) of the velocity projection value $v^{\prime}$ on the $x$ axis: $v^{\prime}=\frac{v}{\cos \alpha}$ will lead to $v^{\prime}=\frac{c \sin \alpha}{\cos \alpha}$.

Using the differential $\mathrm{d} v^{\prime}=\frac{c}{\cos ^{2} \alpha} \mathrm{d} \alpha$ and the differential of the mass $m^{\prime}=m_{0} \cos \alpha \quad$ is $\mathrm{d} m^{\prime}=-m_{0} \sin \alpha \mathrm{d} \alpha$ will lead to the calculation of the energy differential $\mathrm{d} E_{\alpha}=m_{0} \cos \alpha \frac{c \sin \alpha}{\cos \alpha} \frac{c}{\cos ^{2} \alpha} \mathrm{d} \alpha-\frac{c^{2} \sin ^{2} \alpha}{\cos ^{2} \alpha} m_{0} \sin \alpha \mathrm{d} \alpha$ : Finally, using a trigonometric identity leads to $\mathrm{d} E_{\alpha}=m_{0} c^{2} \sin \alpha \mathrm{d} \alpha$.

Since the energy angle needed to bring the body to the speed of light is $\alpha=\pi / 2$, the calculation of the energy needed to bring the mass to the speed of light is:

$$
E_{\alpha=\pi / 2}=\int_{0}^{\pi / 2} m_{0} c^{2} \sin \alpha \mathrm{d} \alpha=m_{0} c^{2}
$$

\subsubsection{One-Dimensional Elastic Collision}

Attention is now focused on calculating a completely elastic collision in accordance with our model. The coefficient of restitution is equal to 1 , meaning that there is no energy loss as a result of the collision. The momentum calculation is based on the equation:

$$
m_{1} \boldsymbol{C}_{1}+m_{2} \boldsymbol{C}_{2}=m_{1} \boldsymbol{C}_{1}^{\prime}+m_{2} \boldsymbol{C}_{2}^{\prime}
$$

wherein $C_{1}$ and $C_{2}$ are the self-speed of light vectors of the two bodies before the collision and $C_{1}^{\prime}$ and $C_{2}^{\prime}$ are the self-speed light vectors of the two bodies after the collision.

Inserting the value of the self-speed of light vectors $C=c \cdot \cos \alpha \cdot \hat{t}+c \cdot \sin \alpha \cdot \hat{x}$ in this formula will give:

$$
\begin{aligned}
& m_{1} \sin \alpha_{1}+m_{2} \sin \alpha_{2}=m_{1} \sin \alpha_{1}^{\prime}+m_{2} \sin \alpha_{2}^{\prime} \\
& m_{1} \cos \alpha_{1}+m_{2} \cos \alpha_{2}=m_{1} \cos \alpha_{1}^{\prime}+m_{2} \cos \alpha_{2}^{\prime}
\end{aligned}
$$

The top equation in this pair of equations is the momentum formula known 
in classical physics as $m_{1} v_{1}+m_{2} v_{2}=m_{1} v_{1}^{\prime}+m_{2} v_{2}^{\prime}$, while the second equation is the momentum equation in the time axis. The self-time energies of the two bodies before and after the collision will be $E_{s t 1}+E_{s t 2}=E_{s t 1}^{\prime}+E_{s t 2}^{\prime}$, so that:

$m_{1} \cos \alpha_{1}+m_{2} \cos \alpha_{2}=m_{1} \cos \alpha_{1}^{\prime}+m_{2} \cos \alpha_{2}^{\prime}$ is obtained, which is the same equation obtained in the last pair of equations. The same formula will be derived from the state kinetic energy conservation law $E_{\alpha_{1}}+E_{\alpha_{2}}=E_{\alpha_{1}^{\prime}}^{\prime}+E_{\alpha_{2}^{\prime}}^{\prime}$ (wherein $\left.E_{\alpha}=m_{0} c^{2}(1-\cos \alpha)\right)$.

\subsubsection{Plastic Collision}

Attention is now referred to the calculation of a plastic collision, in which two bodies collide and merge into one body. The momentum conservation law will be here according to the following equation:

$$
m_{1} \boldsymbol{C}_{1}+m_{2} \boldsymbol{C}_{2}=m_{3} \boldsymbol{C}_{3}
$$

wherein $C_{2}$ and $C_{2}$ are the self-speed light vectors of the two bodies before the collision and $C_{3}$ is the self-speed light vector of the body with mass $m_{3}$ after the collision. Inserting the self-speed light vector value will lead to the following pair of formulas:

$$
\begin{aligned}
& m_{1} \sin \alpha_{1}+m_{2} \sin \alpha_{2}=m_{3} \sin \alpha_{3} \\
& m_{1} \cos \alpha_{1}+m_{2} \cos \alpha_{2}=m_{3} \cos \alpha_{3}
\end{aligned}
$$

The loss of energy $m_{1} c^{2}+m_{2} c^{2}>m_{3} c^{2}$ is part of the mass of the two bodies, the mass $m_{3}$ will always be smaller than the sum of the two masses $m_{1}$ and $m_{2}$ and the difference between them is $\Delta m$ :

$$
\begin{gathered}
m_{1}+m_{2}=m_{3}+\Delta m \\
\Delta m=\left(m_{1}+m_{2}\right)\left(1-\sqrt{1-\frac{4 m_{1} m_{2}}{\left(m_{1}+m_{2}\right)^{2}} \sin ^{2} \frac{\alpha_{1}+\alpha_{2}}{2}}\right)
\end{gathered}
$$

Using formulas $\lim _{x \rightarrow 0}(1-x)^{0.5}=\lim _{x \rightarrow 0}(1-0.5 x)$ and $\lim _{\alpha \rightarrow 0} \sin \alpha=\lim _{\alpha \rightarrow 0} a$ will lead for small speeds to the result:

$$
\Delta m=\frac{m_{1} m_{2}}{2\left(m_{1}+m_{2}\right)}\left(\sin \alpha_{1}-\sin \alpha_{2}\right)^{2}
$$

and the loss of energy at small speeds will be:

$$
\Delta E=\Delta m c^{2}=\frac{m_{1} m_{2}}{2\left(m_{1}+m_{2}\right)}\left(v_{1}-v_{2}\right)^{2}
$$

This is the same result known from classical physics, in a plastic collision, i.e. the loss of energy as a result of the plastic collision $\Delta E$ is equal to the value of the energy loss of mass difference $m$ due to collision $\Delta E=\Delta m c^{2}$.

\subsection{Solving Problems According to the Model}

In this sub-section, it will be shown how this new theory can be applied to solving complicated and difficult current problems in physics, based on this model, which are simpler and easier solutions to understand. Problems as examples in- 
clude the Twin Paradox. The expansion of the universe in acceleration and a black hole, which we will explain in the next chapter.

\subsubsection{The Twin Paradox}

Figure 12 describes a solution to the Twin Paradox in a simple way, by showing the speed of the moving frame in space-time, in two directions. By placing the value $\Delta x^{\prime}=0$ (the traveling brother remains at the origin of the axes of the moving frame) from the equations of Lorentz's transformation obtained in Equation (6), the time difference between the two frames is:

$\Delta T=2 \Delta t\left(1-\frac{1}{\gamma}\right)=2 \frac{\Delta x}{v}\left(1-\frac{1}{\gamma}\right)$. The reference frame is of the brother who is left behind, whose self-speed light vector is $C_{1}=\left(c, \frac{\pi}{2}\right)$. As soon as the twins separate, the self-speed light vector of the moving frame is $C_{2 F}=\left(c, \frac{\pi}{2}-\alpha\right)$, therefore the speed of the moving frame in space-time calculated by Equation (8) is: $\boldsymbol{V}_{a x F}=\boldsymbol{C}_{2 F}-\boldsymbol{C}_{1}=\left(2 c \sin \left(\frac{\alpha}{2}\right),-\frac{\alpha}{2}\right)$. When the traveling twin returns, at the same speed but in the opposite direction, the self-speed light vector is $C_{2 B}=\left(c, \frac{\pi}{2}+\alpha\right)$, therefore the velocity of the frame in space is $\boldsymbol{V}_{a \times B}=\boldsymbol{C}_{2 B}-\boldsymbol{C}_{1}=\left(2 c \sin \left(\frac{\alpha}{2}\right), \pi+\frac{\alpha}{2}\right)$. Using trigonometric identities $2 \cos \frac{\alpha}{2} \sin \frac{\alpha}{2}=\sin \alpha$ and $2 \sin ^{2} \frac{\alpha}{2}=1-\cos \alpha$ will lead to the value of time difference $\Delta T$ by placing it in Equation (3):

$$
\Delta T=2 \frac{\Delta x}{v}(1-\cos \alpha)=2 \frac{\Delta x}{v}\left(1-\frac{1}{\gamma}\right)
$$

Theoretically, we obtain:

$$
\Delta T<2 \frac{\Delta x}{v} \text { for } 0<\alpha<\frac{\pi}{2} \text {, when } 2 \frac{\Delta x}{v} \text { is the time which the stationary twin }
$$
observes. $\Delta T>2 \frac{\Delta x}{v}$ for $\frac{\pi}{2}<\alpha<\pi$, so that the traveling twin seems to be coming back before the beginning of the trip, i.e. movement back in time.

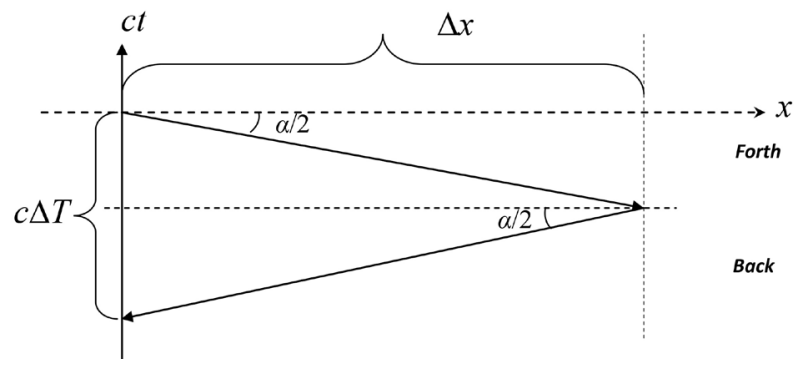

Figure 12. Solving the Twin Paradox by showing the velocity of the frame moving in space-time in two directions, we obtain that the returning twin is younger with a difference of $\Delta T$. 
Figure 13 describes the motion of the moving twin relative to his twin brother in the axial frame (using ICF). The $c t$ axis is at an angle $+\alpha$ relative to the $c t^{\prime}$ axis on the way forth, compared to the way back, when the angle is $-\alpha$. Here as well, we obtain the same time difference between the two frames.

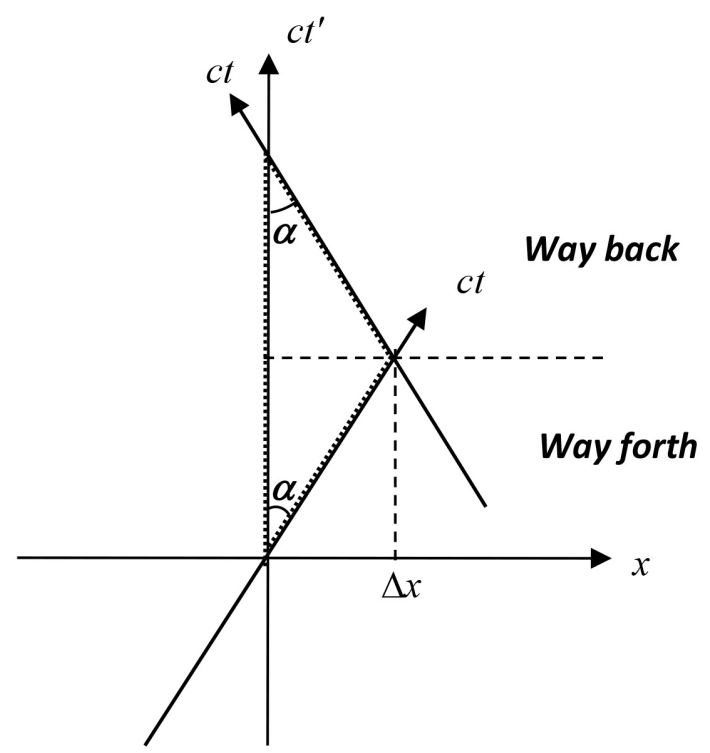

Figure 13. The movement of the traveling brother relative to his twin brother.

\section{Discussion and Conclusions}

\subsection{Comparison with Previous Studies}

Many researchers have examined the issue of negative mass, already in 1951, as part of the Gravity Research Foundation. The possibility of negative mass was tested, and how it would behave under gravitational and also other forces [7]. In 2017, researchers at the University of Washington created an inert experimental negative mass by refrigeration of rubidium atoms with lasers, although this was not an entirely real negative mass [8]. In fact, no research has succeeded yet in practice, neither in experiments nor by adequate formulas, to realize or prove the existence of negative mass. The theory here opens thus a door to a new and complete world in physics which will change the laws of physics by showing how a negative mass can be described using the principles of special relativity.

\subsection{Summary}

It can be seen that the current study provides a solution to a physics problem which encountered nowadays, i.e., that a real body cannot have a negative mass, nor move in a negative space-time. The theory here presents a model which predicts its achievement. Since the model includes a body of a negative mass, forces of repulsion are created between the bodies, and the possibility of creating bodies with negative mass, rejection forces and negative time, which is actually a returning to the past, opens up possibilities and implications in the fields of 
technology, starting from solving problems, and equations in science, as can be seen in the above description, leading to new inventions of products and processes in many industrial fields. The main results and conclusions are:

1) Each body or particle moves at the speed of light in the fourth dimension, which is the time axis. Each body has a self-speed of light vector of the stationary reference frame $\boldsymbol{C}=(c, 0,0,0)$ in the fourth dimension, and a self-speed light vector of the moving Euclidean frame $\boldsymbol{C}^{\prime}=\left(v_{t}^{\prime}, v_{x}^{\prime}, v_{y}^{\prime}, v_{z}^{\prime}\right)$. The body has an energy angle $a$ between the two self-speed light vectors of the stationary reference frame and the moving Euclidean frame. When the energy angle is bigger than $\pi / 2$, the body mass is negative with negative self-time, and a return to the past becomes possible.

2) Repulsion gravitational forces between bodies, besides the regular attraction forces, can be seen.

3) When the body speed increases, its effective mass decreases.

4) The model solves the Twin Paradox in a simple and easy to understand way.

5) The model describes a method for calculating the amount of energy required to bring a body from a rest state to the speed of light.

6) The model describes a way to calculate energy and momentum, such that in the case of a completely elastic collision, there is no loss of energy as a result of the collision.

7) In the case of a plastic collision, it gives that the energy losses are part of the mass of the two unified bodies.

8) When the energy angle $\alpha$ is equal to $\pi / 2$, the speed of the moving body reaches the speed of light and the state kinetic energy is equal to $m_{0} c^{2}$. With greater energy input, the body speed will decrease until the energy angle $\alpha$ reaches $\pi$, then the body will be at rest with state kinetic energy of $2 m_{0} c^{2}$ and self-time energy $\left(-m_{0} c^{2}\right)$.

The continuation of this study:

9) This model may provide an explanation of how the universe is accelerating and expanding and will avoid the need of the assumption of existence of the dark matter and energy in cosmology.

10) The model may describe the black hole equations in a simpler way.

11) The model may provide more precise formulas even for general relativity.

12) The model will aid research on what happens in quantum physics, and may explain, for example the Heisenberg's uncertainty principle.

Future studies may continue to develop the equations presented in this new model, and may provide experimentally creation of negative mass.

\section{Acknowledgements}

To my dear family who believed and supported me for years. To my life teacher Prof. Aharon Peled, who encouraged me and accompanied me all along. Also thanks to Prof. Hashem Zuabi and Dr. David Nir. 


\section{Conflicts of Interest}

The author declares no conflicts of interest regarding the publication of this paper.

\section{References}

[1] Einstein, A. (1905) Zur Elektrodynamik der bewegter Körper. Ann. Phys., 17, 891. eprint in English (1923) The Principle of Relativity. Dover, New York, 35-65.

[2] Galileo, G. (1967) Dialogue Concerning the Chief World Systems: Ptolemaic and Copernican, Eng. Trans. S. Drake, foreword A. Einstein, 2nd Edition, University of California Press, Berkeley.

[3] Lorentz, H. (1904) Proc. Acad. Sci., 6, 809.

[4] Minkowski, H. (1909) Phys. Z., 10, 104.

[5] Rothenstein, B., Popescu, S. and Spix, G.J. (2006) A Brief and Transparent Derivation of Lorentz-Einstein Transformations via Thought Experiments. https://arxiv.org/ftp/physics/papers/0602/0602054.pdf

[6] Minkowski, H. (1908) Space and Time. Cologne Conference, Reprint in English (1923) The Principle of Relativity, Dover, New York, 75-91.

[7] Luttinger, J.M. (1951) On Negative Mass in the Theory of Gravitation. Gravity Research Foundation, University of Wiscosin, Madison, Wis.

[8] Khamehchi, M.A., Hossain, K., Mossman, M.E., Zhang, Y.P., Busch, Th., Forbes, M.M. and Engels, P. (2017) Physical Review Letters, 118, 155301. 